\title{
A Literature Survey ON RECOMMENDATION SYSTEM BASED ON SENTIMENTAL ANALYSIS
}

\author{
Achin Jain $^{1}$, Vanita Jain ${ }^{2}$ and Nidhi Kapoor ${ }^{3}$ \\ BharatiVidyapeeth College of Engineering, New Delhi
}

\begin{abstract}
Recommender systems have grown to be a critical research subject after the emergence of the first paper on collaborative filtering in the Nineties. Despite the fact that educational studies on recommender systems, has extended extensively over the last 10 years, there are deficiencies in the complete literature evaluation and classification of that research. Because of this, we reviewed articles on recommender structures, and then classified those based on sentiment analysis. The articles are categorized into three techniques of recommender system, i.e.; collaborative filtering $(C F)$, content based and context based. We have tried to find out the research papers related to sentimental analysis based recommender system. To classify research done by authors in this field, we have shown different approaches of recommender system based on sentimental analysis with the help of tables. Our studies give statistics, approximately trends in recommender structures research, and gives practitioners and researchers with perception and destiny route on the recommender system using sentimental analysis. We hope that this paper enables all and sundry who is interested in recommender systems research with insight for destiny.
\end{abstract}

\section{KEYWORDS}

Recommender systems; Literature review, Sentimental analysis

\section{INTRODUCTION}

We currently live in an era of information. We are surrounded by a plethora of data in the form of reviews, blogs, papers and comments on various websites. The number of people around the world who use the internet has witnessed an increase of approximately $40 \%$ since 1995 and reached a count of 3.2 billion. The increased information flow has opened more avenues, but it has also led to added confusion for the user. Amidst this huge amount of data, the task of making certain decisions becomes difficult. It is rightly said that one should make an informed decision, but too much information can also hinder the decision-making process. Thus, in order to save a user from this confusion and make the experience of surfing the internet a pleasurable one, recommender systems were introduced. Francesco Ricci, LiorRokach and BrachaShapira define the recommender systems as software tools that make relevant suggestions to a user [1], [2]. Depending upon the user profile and the product profile, which are formed using various techniques and algorithms, suggestions are made. More than $32 \%$ of consumers rate a product online, over $33 \%$ writes reviews and nearly $88 \%$ trust online reviews [14]. Thus, reviews play an essential role in affecting the sales of a commodity or a service. Each review posted on the web consists of the user's sentiments (positive or negative) and preferences. Sentiment analysis helps in determining the attitude of the writer by computationally dividing opinions in a piece of text into positive, negative or neutral [11]. Extracting the sentiments in reviews can largely contribute to the quality of the recommender system by incorporating in it valuable information present in the reviews and also help in the understanding that how a particular review affects the consumer DOI:10.5121/acii.2016.3103 
[11], [13]. Different techniques such as the weighted algorithm are used to produce scores for different texts [13].The usage of the process of sentiment analysis paves the way for the development of personalized recommender system. Extensive research has been done in this field of recommendation systems. Recommendation systems have been broadly divided into three categories. These categories are collaborative filtering, content based and context based recommendation systems.

The rest of the paper is organized as follows: Section 2 gives a brief overview of various types of Recommender systems. Section 3, 4 and 5 gives a detailed overview about the types of recommender systems with related work carried out by researchers in tabular form.Finally, section 6 concludes this paper.

\section{TYPES OF RECOMMENDER SYSTEM}

Over the years, recommender systems have been studied widely and are divided into different categories according to the approach being used. The categories are collaborative filtering $(\mathrm{CF})$, content based and context based.

\subsection{Collaboration filtering}

Collaborative filtering (CF) uses the numerical reviews given by the user and is mainly based upon the historical data of the user available to the system [4], [5]. The historical data available helps to build the user profile and the data available about the item is used to make the item profile. Both the user profile and the item profile are used to make a recommendation system. The Netflix Competition has given much popularity to collaborative filtering [7], [5]. Collaborative filtering is considered the most basic and the easiest method to find recommendations and make predictions regarding the sales of a product. It does have some disadvantages which has led to the development of new methods and techniques.

\subsection{Content Based Recommender System}

Content based [9] systems focus on the features of the products and aim at creating a user profile depending on the previous reviews and also a profile of the item in accordance with the features it provides and the reviews it has received [8], [5].It is observed that reviews usually contain product feature and user opinion in pairs [5], [9], [10]. It is observed that users' reviews contain a feature of the product followed by his/her opinion about the product. Content based recommendation systems help overcome sparsity problem that is faced in collaborative filtering based recommendation system.

\subsection{Context Based Recommender System}

Extending the user/item convention to the circumstances of the user to incorporate the contextual information is what is achieved in context-based recommender systems [15]. This helps to abandon the cumbersome process of making the user fill a huge number of personal details. 


\section{Collaborative Filtering}

Collaboration filtering is a technique for predicting unknown preferences of people by using already known preferences from many users [6]. It computes similarity on two basis: one is user and the other is the item. It uses cosine and Pearson correlation similarity approach [19]. The main challenges that Collaborative Filtering deals with are data sparsity, scalability and cold start problem. CF introduces three main algorithms: memory-based, model-based, and hybrid CF, which are used to combine $\mathrm{CF}$ with other recommendation techniques and their power to deal with the challenges [5].

\section{Cosine similarity}

Given two vectors of attributes, $\mathrm{A}$ and $\mathrm{B}$, the cosine similarity, $\cos (\theta)$, is represented using a dot product and magnitude as[25].

$$
\text { similarity }=\cos (\theta)=\frac{A \cdot B}{\|A\|\|B\|}=\frac{\sum_{i=1}^{n} A_{i} \times B_{i}}{\sqrt{\sum_{i=1}^{n}\left(A_{i}\right)^{2}} \times \sqrt{\sum_{i=1}^{n}\left(B_{i}\right)^{2}}}
$$

\section{Pearson correlation similarity}

$$
\mid \operatorname{Pearson}(x, y)=\frac{\sum x y-\frac{\sum x \sum y}{N}}{\sqrt{\left(\sum x^{2}-\frac{\left(\sum x\right)^{2}}{N}\right)\left(\sum y^{2}-\frac{\left(\sum y\right)^{2}}{N}\right)}}
$$

Where (x, y) refers to the data objects and $\mathrm{N}$ is the total number of attributes [25].

\subsection{Memory-Based Collaborative Filtering (Neighborhood Based)}

People with similar interests are combined to form a group and every user is a part of that group [36]. User -based CF and Item-based CF are used to represent memory-based CF. It is easy to implement and scales well with correlated items. There is no need of considering the content of items being recommended. There are many limitations of memory-based CF like cold start problem, sparsity and their dependencies on human ratings [37].

\subsection{Model-based CF}

Complex patters which are based on training data, are recognized by designing and developing the models (such as data mining algorithms, machine learning) and then intelligent predictions are made for CF tasks for the real world data which are based on learnt models[38,39]. It gives an intuitive rationale for recommendations. Model-building is an expensive procedure. Other disadvantage of model-based $\mathrm{CF}$ is that it loses useful information for dimensionality reduction techniques [37]. 


\subsection{Hybrid Collaborative Filtering Techniques}

In hybrid recommender system, different techniques of collaborative approaches and other recommender techniques (usually content based approaches), combined to get better results. Various problems like cold-start, data sparsity and scalability can be avoided by using hybrid approach [40].There are different ways of combining CF with other recommender techniques which are following:

- Hybrid Recommenders Incorporating CF and Content-Based Features

- Hybrid Recommenders Combining CF and Other Recommender Systems

- Hybrid Recommenders Combining CF Algorithms [37].

Fig. 1. illustrates the flow chart of Collaborative Filtering Recommender System. It shows how collaborative filtering considers only numerical reviews given by different users and then gives recommended products as result. The user reviews are stored in a database to make further refrences and predictions. In the figure User 1 and User 6 show similar behaviour and thus their profiles lie in the same neighborhood which indicates similar interests. Using this similarity, review about a product not rated by User 6 can be predicted using the reviews of User 1 that are available. Thus, a prediction regarding product C's review by User 6 is made using the available data. From these predictions, recommendations are extracted and suggested to the user.

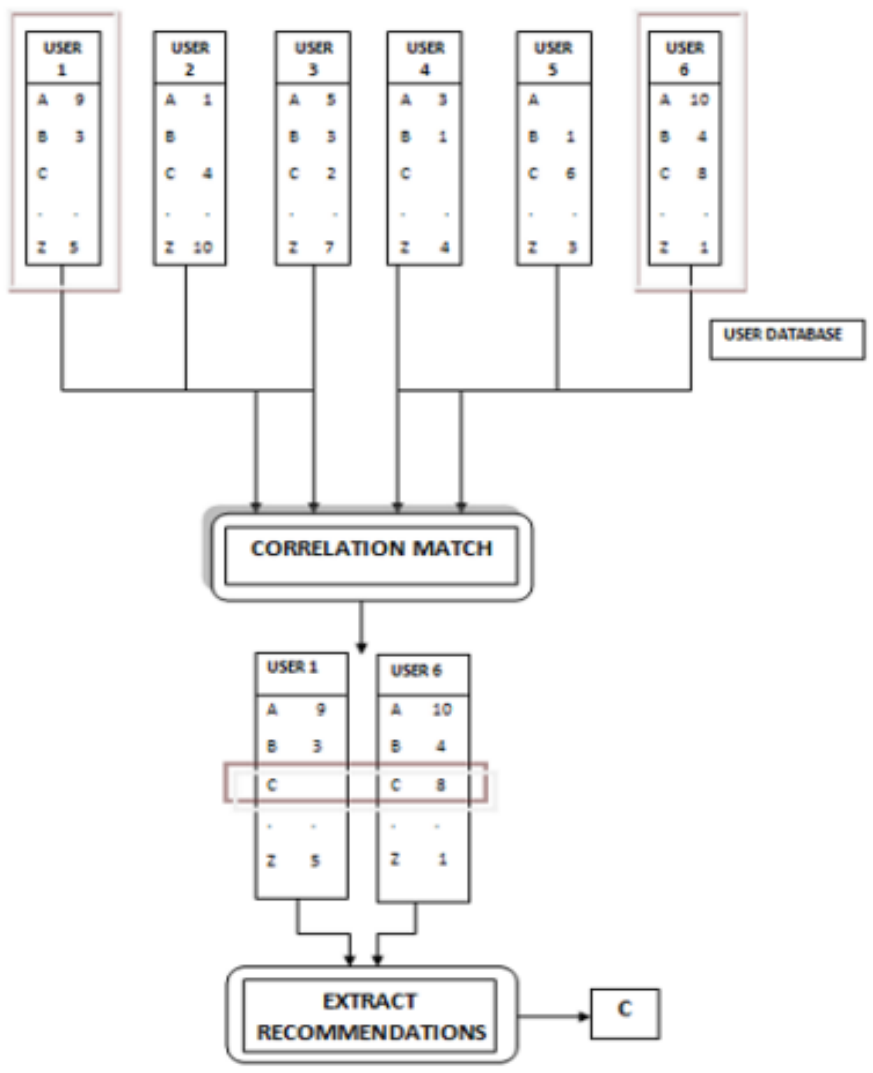

Figure 1. Collaborative Filtering Recommender System 
Table 1 shows different collaborative filering methods based on sentimental analysis for recommendation system. These methods are based on a variety of different models. Over the yaers, different models are combined with collaborative filtering technique of recommender systems in order to increase the accuracy of the predictions given by the results. J. Wang et. Al. developed the unified relevance model based recommendation system while Parlov et. Al. used maximum entropy approach and both these models helped reduce data spartsity problem, one of the commonly faced problem in collaborative filtering based recommendation systems.

Table 1. Collaborative Filtering Methods

\begin{tabular}{|l|l|l|}
\hline Method & Description & References \\
\hline $\begin{array}{l}\text { Unified relevance } \\
\text { model }\end{array}$ & $\begin{array}{l}\text { It is a probabilistic item-to-user relevance } \\
\text { framework which uses Parzen-window } \\
\text { method for density estimation. This approach } \\
\text { reduces data sparsity problem. }\end{array}$ & $\begin{array}{l}\text { J. Wang, A. P. de } \\
\text { Vries, and M. J. T. } \\
\text { Reinders[28] }\end{array}$ \\
\hline Hybrid CF model & $\begin{array}{l}\text { It introduces effective recommender system } \\
\text { using sequential mixture CF and joint mixture } \\
\text { CF. It also implements advanced Bayes belief } \\
\text { networks. }\end{array}$ & $\begin{array}{l}\text { X. Su, R. Greiner, } \\
\text { T. M. } \\
\text { Khoshgoftaar[27] }\end{array}$ \\
\hline $\begin{array}{l}\text { Fuzzy Association } \\
\text { Rules and } \\
\text { Multilevel }\end{array}$ & $\begin{array}{l}\text { It uses fuzzy association rule mining to extend } \\
\text { the existing techniques. FARAMS achieved the } \\
\text { tFARAMS) }\end{array}$ & $\begin{array}{l}\text { C. W. K. Leung, S. } \\
\text { C. F. Chan, and F. } \\
\text { L. Chung[29] }\end{array}$ \\
\hline $\begin{array}{l}\text { Flexible mixture } \\
\text { model (FMM) }\end{array}$ & $\begin{array}{l}\text { Simultaneous creation of user and item clusters. } \\
\text { It introduces preference nodes to study a } \\
\text { dramatic variation of the rating among users } \\
\text { with similar tastes. }\end{array}$ & L. Si and R. Jin[26] \\
\hline $\begin{array}{l}\text { Maximum entropy } \\
\text { approach }\end{array}$ & $\begin{array}{l}\text { Clustering of items based on user access path in } \\
\text { order to reduce the apriori probability. This } \\
\text { helps in addressing sparsity and dimensionality } \\
\text { reduction. }\end{array}$ & $\begin{array}{l}\text { D. Y. Pavlov and } \\
\text { D. M. Pennock } \\
\text { [30] }\end{array}$ \\
\hline
\end{tabular}

\section{CONTENT-BASED RECOMMENDER SYSTEM}

It is a technique where individual user profiles are taken into account. It enhances the user's interest and predicts whether the user would be interested in eating at any particular restaurant or interested in seeing any particular movie [22]. It is also known as adaptive Filtering as it provides suggestions according to user's field of interest and adapts user's likes and dislikes. It represents the comparison between the content contained in the item with the content of items of user's interest. By using Bayesian hierarchical model, better user profiles for upcoming users is made by collecting feedbacks from the old users [20]. Content based collaborative filtering is more widely used to compare pure CF and pure Content-base. In CF the problem of sparsity is overcome (converting sparse user filled matrix into full user rating matrix) by using content-based prediction [21]. Fig.2 displays the flow of information in a content based recommendation system. Relevant entities of an item and relations are kept together as input. Main features of items are extracted from item ontology. Features of items, user's ratings and user modeling data 
are applied to content based recommender system. After applying, various recommended items are given as output.

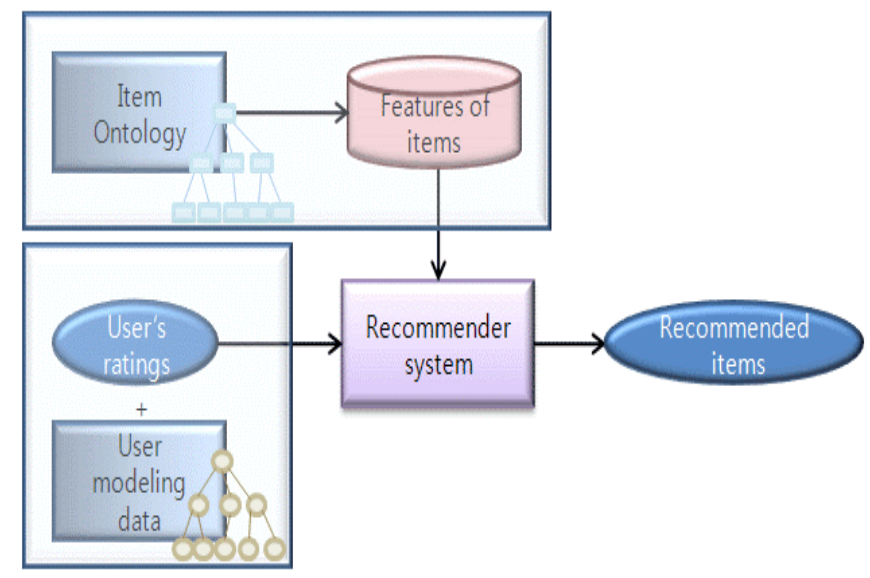

Figure 2. Content-Based Recommender System

Table 2 shows different content based methods using sentimental analysis for recommendation system. These methods are based on a variety of different models. Different researchers have studied the relevance of various techniques that can be implemented in content based recommendation systems. Accuracy and relevance of the recommendation systems have become better by extensive research. Content-Boosted Collaborative filtering technique was implemented by Prem Melville et. Al. Recommendation system using content based technique and Bayesian Hierarchical Model (BHM) was built by Marko Balabanovic.

Table 2. Content-Based Recommender System

\begin{tabular}{|l|l|l|}
\hline Method & Description & References \\
\hline $\begin{array}{l}\text { Content-Boosted } \\
\text { Collaborative Filtering }\end{array}$ & $\begin{array}{l}\text { It gives an approach to combine } \\
\text { content and collaboration to enhance } \\
\text { existing user data and to give better } \\
\text { performance than a pure content } \\
\text { based predictor. }\end{array}$ & $\begin{array}{l}\text { Prem Melville, Raymond } \\
\text { Roney, }\end{array}$ \\
\hline FAB Technique & $\begin{array}{l}\text { An adaptive recommendation service } \\
\text { for collection and selection of web } \\
\text { pages. It makes the system more } \\
\text { personalized and combines the } \\
\text { benefits of content analysis with the } \\
\text { shared user interests. }\end{array}$ & \\
\hline $\begin{array}{l}\text { Bayesian } \\
\text { hierarchical } \\
\text { model(BHM) }\end{array}$ & $\begin{array}{l}\text { Proposes a faster technique to gather } \\
\text { a huge number of individual user } \\
\text { profiles even if feedbacks available } \\
\text { are less. It uses various parameters of } \\
\text { BHM for optimization of joint data } \\
\text { likelihood. }\end{array}$ & $\begin{array}{l}\text { Koren } \\
\text { [20] }\end{array}$ \\
\hline
\end{tabular}




\begin{tabular}{|l|l|l|l|}
\hline Keyword & Map & $\begin{array}{l}\text { It captures the relations between } \\
\text { conditions that the learner has been } \\
\text { exposed to, which are used to }\end{array}$ & $\begin{array}{l}\text { Rubens, Toshio Okamoto } \\
\text { and Yan Feng }\end{array}$ \\
represent the knowledge of the & [23] \\
learner. The relevance and & \\
complement of learning resources & \\
recommendation are increased by & \\
keyword map. It is better suited for & \\
e-learning settings and achieves \\
higher accuracy than common \\
recommender methods.
\end{tabular}

\section{CONTEXT-BASEd RECOMMENDER SYSTEM}

Recommender systems which use collaborative filtering technique or which are based on the contents and features of items have achieved great success but can be further improved. The Higher level of personalization is required in recommender systems to give more appropriate suggestions. In order to achieve this, the contextual information of users is also taken into consideration while designing a recommender system. Context refers to the time, location, area and environment of the user which define a user's status. Incorporating contextual information in a recommender system helps to get a clear picture of the situation of any individual, place or object which is of relevance to the system for prediction [17], [18].

It aids in extracting information about a particular community of individuals and this information proves to be of high importance to improve the suggestions provided to a user and makes the system more efficient. Recommender systems require situational information of the user and context based recommender system accesses this information directly using various techniques (such as GPS) [16] and does not bother the user with this. Fig. 3 displays the flow of contextual information in a context based recommender system. The user's location data, social data, current time, weather data is taken into consideration as the contextual data and is given as input to the system. An approximate address of the user is determined and the location is saved. Social data of a user can be accessed by requesting permission to a social account of the user.

The device the person is using can be used to get the time of the day which play a role in finding suitable recommendations for the user. Accessing the location can also help in finding the weather of the place. All the information gathered is processed in a system and sentiment analysis is done. After processing the system gives an output of a list of attractions for the user. Depending on the application of the system, desired results are produced. If the application is for a tourist, destinations or shortest routes or hotels might be given as recommendations.

Contextual factors are of two types: Dynamic and static, depending on whether they change with time or not. .

1. Dynamic: When the contextual factors change over time and hence unstable. They may change by explicit user feedback. User feedback is generally used for refining the profile of user to get better results of recommendations. The biggest challenge is that if a system is considered to be dynamic then the system should be able to find out when to switch to a different underlying context model. 
2. Static: The contextual factors don't change over time and hence stable. For e.g. to buy a cell phone the contextual factors can be Time, purpose of purchasing and only them while entire purchasing purpose recommendation application runs.

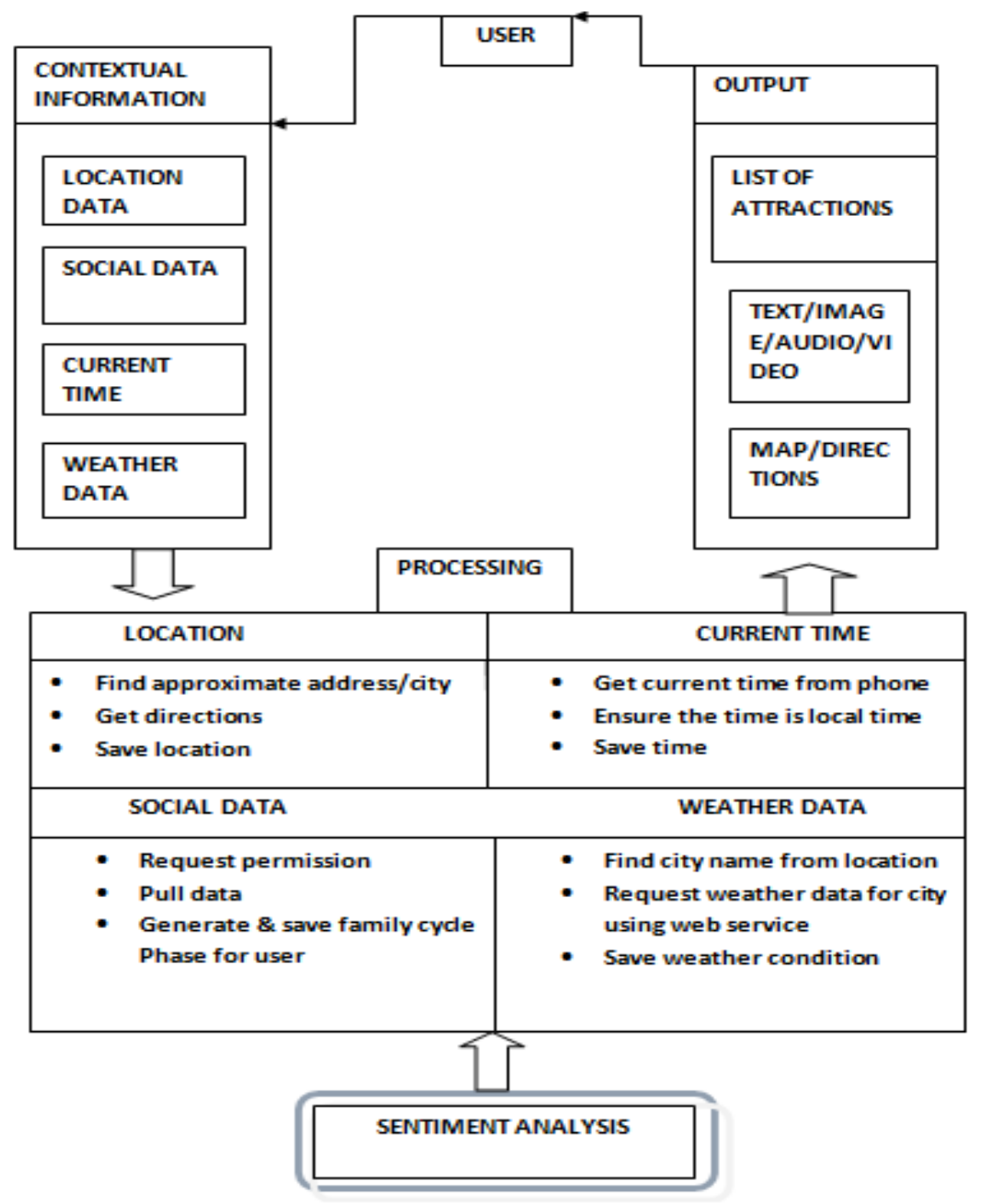

Figure 3. Context-Based Recommender System

Contextual factors are of three types: Fully observable, partially observable and Unobservable, depending on what is being observed (or what system exactly knows).

1. Fully observable: Complete structure and values of contextual factors are known explicitly, at the time when recommendations are made.

2. Partially observable: Some of the information is known explicitly about the contextual factors.

3. Unobservable: There is no information of contextual factors explicitly available in it.[34]. 


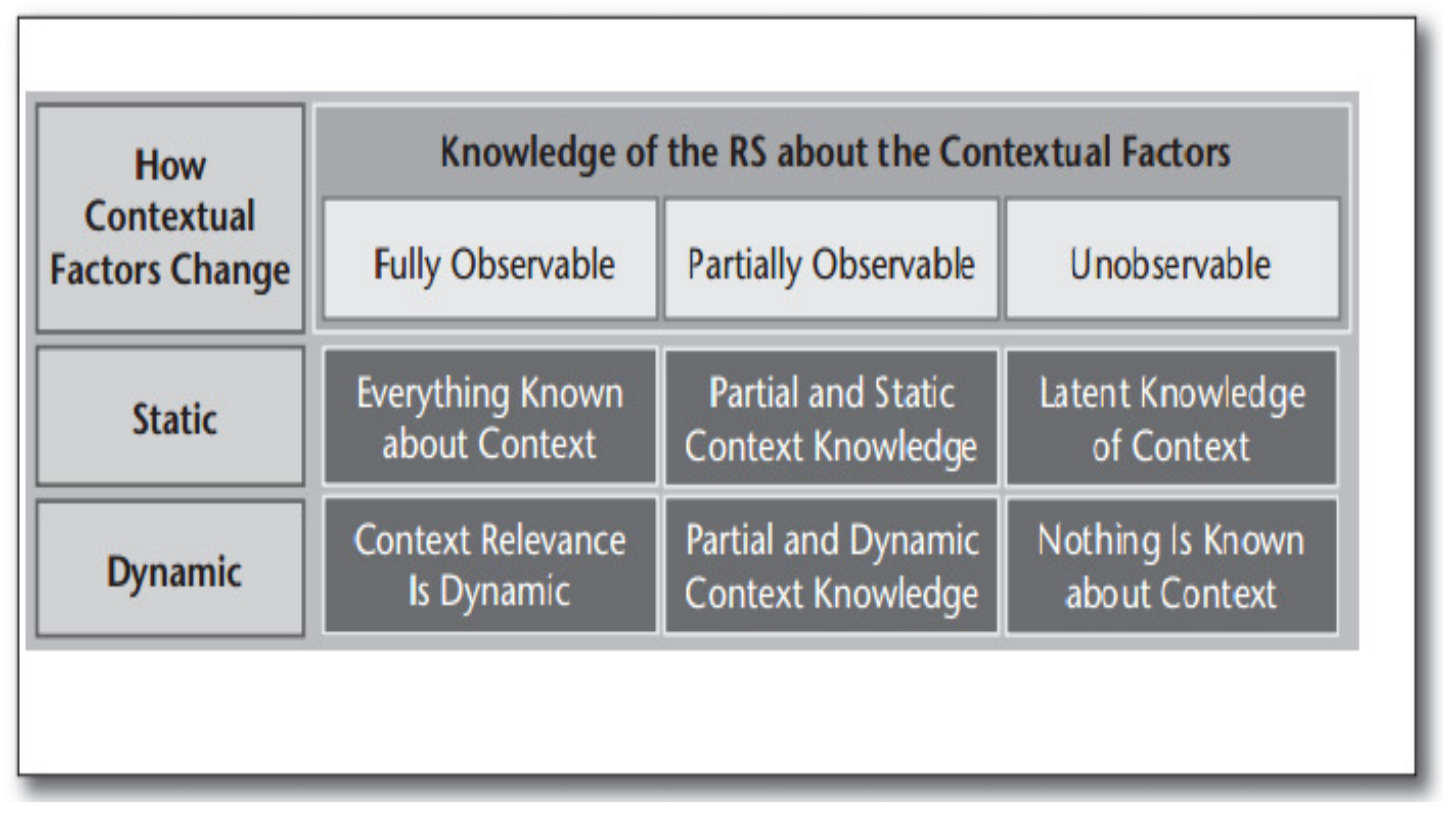

Figure 4. Contextual Information Dimensions

Table 3 shows different context based methods with sentimental analysis for recommendation system. The table gives recommender system by Norma Saiph Savage in which context based technique using Hidden Markov Model is used. It helps improve location recommendations.Maciej Baranski et al. used a multidimensional database to help increase credibility of a system. Gediminaset. Al. used Fuzzy Bayesian Networks. . Human memory model was used as the base for a context based system by Sarabjot Singh Anand et al. VíctorCodina et al. created a recommendation system based on Matrix-factorization Predictive Context Model and observed more accurate results.

Table 3. Context-Based Recommender System

\begin{tabular}{|c|c|c|}
\hline METHOD & DESCRIPTION & REFERENCES \\
\hline Hidden Markov Model & $\begin{array}{l}\text { Improved version of a location } \\
\text { recommender system by } \\
\text { implementing Decision Tree (DT) } \\
\text { along with discrete Hidden Markov } \\
\text { Model (HMM).Together HMM and } \\
\text { DT differentiate between transport } \\
\text { modes and reduce noise. }\end{array}$ & $\begin{array}{l}\text { Norma Saiph Savage } \\
\text { Maciej Baranski } \\
\text { Norma Elva Chavez } \\
\text { Tobias Höllerer[34] }\end{array}$ \\
\hline $\begin{array}{l}\text { Multidimensional } \\
\text { approach }\end{array}$ & $\begin{array}{l}\text { It provides additional contextual } \\
\text { information on user and item, and } \\
\text { also supports multiple } \\
\text { Dimensions, profiling information, } \\
\text { and hierarchical aggregation of } \\
\text { recommender system. }\end{array}$ & $\begin{array}{l}\text { GediminasAdomavicius } \\
\text { Ramesh } \\
\text { Sankaranarayanan } \\
\text { Shahana Sen } \\
\text { Alexander Tuzhilin } \\
\text { [31] }\end{array}$ \\
\hline $\begin{array}{ll}\text { Fuzzy } & \text { Bayesian } \\
\text { Networks } & \\
\end{array}$ & $\begin{array}{l}\text { It gives a recommender system } \\
\text { which exploits the fuzzy system, }\end{array}$ & $\begin{array}{l}\text { Han-SaemPark,Ji-Oh } \\
\text { YooSung-Bae Cho }\end{array}$ \\
\hline
\end{tabular}




\begin{tabular}{|l|l|l|}
\hline & $\begin{array}{l}\text { Bayesian Networks in order to get } \\
\text { appropriate recommendation with } \\
\text { respect to the context. }\end{array}$ & \\
\hline Human memory model & $\begin{array}{l}\text { A recommender system is proposed } \\
\text { which retrieves relevant preference } \\
\text { information from long term } \\
\text { memory and uses } \\
\text { it in conjunction with the } \\
\text { information stored in short term } \\
\text { memory. }\end{array}$ & $\begin{array}{l}\text { Sarabjot Singh Anand } \\
\text { and BamshadMobasher } \\
{[32]}\end{array}$ \\
\hline $\begin{array}{l}\text { Matrix-factorization } \\
\text { Predictive Context }\end{array}$ & $\begin{array}{l}\text { Distributional-Semantics Pre- } \\
\text { filtering (DSPF) approach is used } \\
\text { to build more precise context aware } \\
\text { Model }\end{array}$ & $\begin{array}{l}\text { Víctor Codina } \\
\text { rating prediction models, by } \\
\text { exploiting, in a novel way, the } \\
\text { distributional Semantics of } \\
\text { contextual conditions. It also shows } \\
\text { how DSPF can be improved by } \\
\text { using clustering techniques. }\end{array}$ \\
[35] \\
\hline
\end{tabular}

\section{Conclusions}

Recommender structures are proving to be a useful device for addressing a part of the records overload phenomenon from the internet. Its evolution has followed the evolution of the internet. The primary technology of recommender system used conventional web sites to gather information from the following sources: (a) content material-primarily based records (b) demographic statistics, and (c) memory-primarily based information.

Latest research shows the use of Sentimental Analysis in developing of more accurate recommender system. These types of methods are commonly used in e-commerce business. In this paper we have classify various approaches of recommender system that are based on Sentiemental analysis.

Future research will deal with advancing the prevailingtechniques and algorithms to enhance the nice of recommenderstructures predictions and hints.

\section{REFERENCES}

[1] Francesco Ricci, LiorRokach, BrachaShapira and Paul B. Kantor - Recommender Systems Handbook; First Edition; Springer-Verlag New York, Inc. New York, NY, USA, 2010.

[2] Tariq Mahmood and Francesco Ricci," Improving recommender systems with adaptive conversational strategies", $20^{\text {th }}$ ACM conference on Hypertext and Hypermedia, pp. 73-82, ACM, July 2009.

[3] Tariq Mahmood, Francesco Ricci, Adriano Venturini and Wolfram Höpken, "Adaptive recommender systems for travel planning", Information and Communication Technologies in Tourism 2008, Proceedings of the International Conference,Innsbruck Austria, pp. 1 - 11, 2008.

[4] X. Su and T. Khoshgoftaar, "A survey of collaborative filtering techniques," Advances in Artificial Intelligence, vol. 2009, pp. 19, August 2009. 
[5] Yongfeng Zhang, Min Zhang and Yiqun Liu, "Incorporating Phrase-level Sentiment Analysis on Textual Reviews for Personalized Recommendation",Eighth ACM International Conference on Web Search and Data Mining, pp. 435 - 440, February 2015.

[6] P. Resnick and H. R. Varian, "Recommender systems," Communications of the ACM, vol. 40, no. 3, pp. 56-58, 1997.

[7] J. Bennett and S. Lanning, "The Netflix Prize", ACM SIGKDD Explorations Newsletter - Special issue on visual analytics, Vol. 9 Issue 2, pp. 51 - 52, December 2007.

[8] Titov and R. McDonald, "A joint model of text and aspect ratings for sentiment summarization", Annual Meeting of the Association for Computational Linguistics, pp. 308 - 316, June 2008.

[9] P. Lops, M. de Gemmis and G. Semeraro, "Content-based recommender systems: State of the art and trends", Recommender Systems Handbook, pp. 73 - 105, 2011.

[10] X. Ding, B. Liu, and P. S. Yu, "A Holistic Lexicon-Based Approach to Opinion Mining", Web Search and Data Mining, pp. 231 - 239, February 2008.

[11] A. Kennedy and D. Inkpen, "Sentiment Classification of Movie and Product Reviews Using Contextual Valence Shifters", Computational Intelligence, Vol. 22, No. 2, pp. 110 - 125, May 2006.

[12] A. Andreevskaia, S. Bergler and M. Urseanu "All Blogs Are Not Made Equal: Exploring Genre Differences in Sentiment Tagging of Blogs", International Conference on Weblogs and Social Media (ICWSM-2007), Boulder, CO, March 2007.

[13] AntonisKoukourikos, GiannisStoitsis and Pythagoras Karampiperis, "Sentiment Analysis: A tool for Rating Attribution to Content in Recommender Systems", $7^{\text {th }}$ European Conference on Technology Enhanced Learning, September 2012.

[14] Bo Pang and Lillian Lee, "Opinion Mining and Sentiment Analysis", Foundations and Trends in Information Retrieval, Vol. 2, Issue 1 - 2, pp. 1 - 135, January 2008.

[15] Umberto Panniello, Alexander Tuzhilin, Michele Gorgoglione, CosimoPalmisano and AntoPedone, "Experimental Comparison of Pre- vs. Post-Filtering approaches in Context-Aware Recommender Systems", ACM Conference on Recommender Systems, October 2009.

[16] Fan Yang and Zhi-Meiwang, "A Mobile Location-based Information Recommendation System Based on GPS and WEB2.0 Services", WSEAS Transactions on Computers, Vol. 8, Issue 4, pp. 725 - 734, April 2009.

[17] Jae Sik Lee and Jin Chun Lee, "Context Awareness by Case-Based Reasoning in a Music Recommendation System”, UCS, Vol. 4836, pp. 45 - 58, 2007.

[18] Asher Levi, Osnat (Ossi) Mokryn, Christophe Diot and Nina Taft, "Finding a Needle in a Haystack of Reviews: Cold Start Context-Based Hotel Recommender System",6th ACM conference on Recommender Systems, September 2012.

[19] Hyung Jun Ahn, "A new similarity measure for collaborative filtering to alleviate the new user cold-starting problem", Information Sciences: an International Journal, Vol. 178, Issue 1, pp 37 51, January 2008.

[20] Yi Zhang and Jonathan Koren, "Efficient Bayesian Hierarchical User Modeling for Recommendation Systems",30th annual International ACM SIGIR Conference on Research and Development, pp. 47 - 54, July 2007.

[21] Prem Melville, Raymond J. Mooney and RamadassNagarajan, "Content-Boosted Collaborative Filtering for Improved Recommendations", $18^{\text {th }}$ National Conference for Artificial Intelligence (AAAI), pp. 187 - 192, August 2002.

[22] ChumkiBasu, Haym Hirsh and William Cohen, "Recommendation as Classification: Using Social and Content-Based Information in Recommendation", American Association for Artificial Intelligence (AAAI), 1998.

[23] Xin Wan, Neil Rubens, Toshio Okamoto and Yan Feng, "Content Filtering Based on Keyword Map", 2nd International Conference on Electrical, Computer Engineering and Electronics (ICECEE ), pp. 484 - 489, May 2015.

[24] Marko Balabanovic, "An Adaptive Web Page Recommendation Service", First International Conference on Autonomous Agents, pp. 378 - 385, February 1997. 
[25] Paul Marx - Providing Actionable Recommendations: A Movie Recommendation Algorithm with Explanatory Capability, Joseph EulVerlag, 2013.

[26] L. Si and R. Jin, "Flexible mixture model for collaborative filtering," 20th International Conference on Machine Learning, vol. 2, pp. 704-711, August 2003.

[27] X. Su, R. Greiner, T. M. Khoshgoftaar and X. Zhu, "Hybrid collaborative filtering algorithms using a mixture of experts," IEEE/WIC/ACM International Conference on Web Intelligence, pp. 645-649, November 2007.

[28] J. Wang, A. P. de Vries, and M. J. T. Reinders, "Unified relevance models for rating prediction in collaborative filtering," ACM Transactions on Information Systems, vol. 26, no. 3, pp. 1-42, June 2008.

[29] W. K. Leung, S. C. F. Chan, and F. L. Chung, "A collaborative filtering framework based on fuzzy association rules and multi-level similarity,"Knowledge and Information Systems, vol. 10, no. 3, pp. 357-381, 2006.

[30] D. Y. Pavlov and D. M. Pennock, "A maximum entropy approach to collaborative filtering in dynamic, sparse, high-dimensional domains," Neural Information Processing Systems, pp. 14411448, MIT Press, 2002.

[31] GeminasAdomavicius, Ramesh Sankaranarayanan, Shahana Sen and Alexander Tuzhilin, "Incorporating Contextual Information in Recommender Systems Using a Multidimensional Approach", ACM Transactions on Information Systems, Vol. 23, No. 1, pp. 103 - 145, January 2005.

[32] Sarabjot Singh Anand and BamshadMobasher -From Web to Social Web: Discovering and Deploying User and Content Profiles - Contextual Recommendation,Springer-Verlag Berlin, Heidelberg, 2007.

[33] Han-Saem Park, Ji-Oh Yoo, and Sung-Bae Cho, "A Context-Aware Music Recommendation System Using Fuzzy Bayesian Networks with Utility Theory", $3^{\text {rd }}$ International Conference on Fuzzy Systems and Knowledge Discovery, pp. 970-979, September 2006.

[34] Norma Saiph Savage, MaciejBaranski, Norma Elva Chavez and Tobias Höllerer, "Advances in Location-Based Services I'm feeling LoCo: A Location Based Context Aware Recommendation System", Springer, USA, 2011.

[35] Victor Codina, Francesco Ricci and Luigi Ceccaroni, "Distributional Semantic Pre-filtering in Context-Aware Recommender Systems", $3^{\text {rd }}$ ACM Conference on Recommender Systems, pp. 265 $268,2015$.

[36] B. M. Sarwar, G. Karypis, J. A. Konstan, and J. Riedl, "Itembased collaborative filtering recommendation algorithms," in Proceedings of the 10th International Conference on World Wide Web (WWW '01), pp. 285-295, May 2001.

[37] Xiaoyuan Su and Taghi M. Khoshgoftaar," A Survey of Collaborative Filtering Techniques," Department of Computer Science and Engineering, Florida Atlantic University, 777 Glades Road, Boca Raton, FL 33431, USA, August 2009.

[38] J. Breese, D. Heckerman, and C. Kadie, "Empirical analysis of predictive algorithms for collaborative filtering," in Proceedings of the 14th Conference on Uncertainty in Artificial Intelligence (UAI'98), 1998.

[39] C. Basu, H. Hirsh, and W. Cohen, "Recommendation as classification: using social and contentbased information in recommendation," in Proceedings of the 15th National Conference on Artificial Intelligence (AAAI '98), pp. 714-720, Madison, Wis, USA, July 1998.

[40] G. Adomavicius and A. Tuzhilin, "Toward the next generation of recommender systems: A survey of the state-of-the-artand possible extensions," IEEE Trans. on Knowledge and Data Eng., vol. 17, pp. 734-749, June 2005. 